\title{
Special issue on the technologies and applications of big data
}

\author{
V. Neelanarayanan ${ }^{1} \cdot$ V. Vijayakumar ${ }^{2} \cdot{\text { Ron } \text { Doyle }^{3} \cdot \text { Imad Fakhri Taha Alyaseen }}^{4} \cdot$ Sven $_{\text {Groppe }}^{5}$
}

Accepted: 13 September 2021 / Published online: 8 October 2021

(C) The Author(s), under exclusive licence to Springer Science+Business Media, LLC, part of Springer Nature 2021

Big data represents the collection of data characterized by volume, variety, velocity, value, veracity and variability. These characters make it difficult to apply traditional data processing techniques for insight extraction. Bid data serves as a source for confident decision making in industry and society at large. Thus, research in technologies relevant to big data receives increasing attention from not only academia and industry but also from practitioners and governments.

The applications of big data technologies have far reaching implications in various industries such as agriculture, manufacturing, healthcare, transportation, education and so on. The aim of this special issue is to present high quality research articles from academia and industry on the recent advances in different aspects of big data

\footnotetext{
V. Neelanarayanan

neelanarayanan.v@vit.ac.in

V. Vijayakumar

v.varadarajan@unsw.edu.au

Ron Doyle

doyler@wfu.edu

Imad Fakhri Taha Alyaseen

imadf@iium.edu.my

Sven Groppe

groppe@ifis.uni-luebeck.de
}

1 Department of Cyber Physical Systems, School of Computer Science and Engineering, VIT University, VandalurKelambakkam Road, Chennai 600 127, India

2 School of Computer Science and Engineering, University of New South Wales, Sydney, NSW 2052, Australia

3 Computer Science and Entrepreneurship, Wake Forest University, 1834 Wake Forest Rd., Reynolda Hall, Suite 230, Winston-Salem, NC 27106, USA

4 IUM Gombak Campus, Kulliyyah of Information and Communication Technology, 53100 Jalan Gombak, Selangor, Malaysia

5 Institute of Information Systems (IFIS), University of Lübeck, Ratzeburger Allee 160 (Building 64, 2nd Level), 23538 Lübeck, Germany technologies and applications. These articles have been selected after a rigorous review process.

Weather forecasting finds its application in air traffic, marine, agriculture, forestry, utility companies, military application and general public. However, in most part of the world statistical and numerical analysis techniques are used. Therefore, the first paper [1] presents machine learning based approach for weather forecasting. Compared to traditional approach, the proposed approach using machine learning has higher accuracy.

Image segmentation in image analytics is used for pattern detection, photo montage and object recognition application. Image acquisition in under water is a challenge and in general produce low quality images. Therefore, the next paper [2] presents segmentation procedure for underwater images. The algorithm improves color correction and contrast enhancement compared to other existing algorithms.

Social network consists of a set of social actors and social interactions between actors. For information retrieval and interaction identification of influential node is essential. Existing graph-based methods consider only numeric measures for keyword extraction. The next paper [3] presents Semantic graph-based Keyword Extraction Method (SKEM) for ranking. The performance of the proposed SKEM model is validated with real-time tweets of Twitter API.

Search engine algorithm consists of a set of rules that a search engine uses to rank listings in response to a search query. For example, Google uses 'Page Rank Algorithm' for its searches. The next paper [4] proposes a more efficient way of searching the web and better organizing the search result based on the categories of the pages.

One of the main concerns in using cloud technology is preserving security and privacy. The secure processing of personal data in the cloud represents a huge challenge. Most of the existing security controls in cloud computing are not suitable for mobile cloud computing. Therefore, the next article [5] presents the design of a trivial information relaying scheme (TIRS) for mobile cloud computing. The 
proposed design has reduced overhead compared to other approaches in mobile cloud environment.

The next paper [6] presents the implementation of a framework for Distributed Denial of Service (DDoS) attack detection for cloud environment. The framework has been implemented using OpenStack cloud environment. The authors have used real-time data set to prove its efficiency compared to existing solutions.

Internet of Things (IoT) - network of physical objects find wide applications in agriculture, transport, home, healthcare, etc. Safeguarding connected devices and networks in the internet of things (IoT) has led to serious security threats. Medical device used in the hospital severe security vulnerability. Therefore the next paper [7] presents risk assessment technique for security attacks on the Internet of Medical Things (IoMT). The technique helps to identify the highest impact causing security attacks in IoMT.

In the refinement of healthcare practices, interrelated visitation and excessive medications rates are vital. Hence, the next paper [8] extracts pertinent data evidence in a novel manner using a new bivariate probability model and probes their balancing nature.

Greenhouse gases trap heat and make the planet warmer. Therefore, governments are committing themselves to reduce carbon emissions to acceptable levels. Micro Grid (MG) is one of the possible solutions to reduce carbon emission. Therefore, the next paper [9] identifies the limitations of existing Micro Grids and proposes Smart Micro Grid based on integration of modern ICT technologies such as sensors, Fog and Cloud computing etc., to overcome the limitations. The paper presents first of its kind Fog Micro Data Center (fogMDC) powered by micro grids and controlled by Software Defined Networks (SDN). The authors concentrate on performance modeling and economic modeling to guarantee QoS for time sensitive IoT applications. The proposed solution has been evaluated using real time meteorological data.

According to International Federation of Global \& Green ICT "IFGICT", green computing is the study and practice of environmentally sustainable computing or IT. The next paper [10] presents models and load balancing techniques for green computing. The authors show that their approach reduces carbon footprint. A container is a standard unit of software that packages up code and all its dependencies so the application runs quickly and reliably from one computing environment to another. The authors propose containers over virtual machine for reducing carbon emission.

The decentralized type of networks mobile ad hoc network (MANET) faces challenges in load balancing and energy efficiency. Inspired from behavior of ants in colonies, the next article [11] proposes to compute the optimal path. The authors propose Ant-based Efficient Energy and Balanced Routing (A-EEBR) technique for choosing next hop using metrics such as delay, energy drain rate, congestion and link quality. The proposed new approach performs better compared to existing Ant-based Energy Saving Routing (A-ESR). This paper also addresses energy efficiency for reducing carbon foot print.

A vehicular ad hoc network (VANET) consists of groups of moving or stationary vehicles connected by a wireless network. VANET forms the backbone for efficiently communicating among the vehicles. However, VANETs do not have a stable topology. The next article [12] proposes dynamic routing protocol considering Quality of Service (QoS) needs and topology changes. The proposed algorithm has improved packet delivery ration, mean routing load and end-to-end delay.

Context-aware adaptive systems adjust themselves in response to the changes in the operating environment, namely the context at runtime. Correctness of the adaptive systems is ensured using adaptive rules. These adaptive rules must be complete and consistent even when the system experiences unforeseen environmental challenges. Therefore, the next paper [13] presents technique for generation of adaptive rules. The proposed technique generates new adaption rules even in undefined context situation.

Equivalence Class Clustering and bottom-up Lattice Traversal (ECLAT) algorithm is an association rule mining technique. The next paper [14] presents an enhanced version of ECLAT for selecting best cloud service provider by cloud service brokers. The cloud service providers can be ranked based on their past performance using the Enhanced ELCAT (EELCAT) algorithm.

Scheduling is one of the core issues in cloud computing for efficient utilization of resources. Conventional scheduling techniques do not minimize Service Level Agreement (SLA) violations, resource wastage and energy consumption. Therefore, the next paper [15] presents multi objective scheduling algorithm. The proposed Nature-inspired Meta-heuristic Threshold based Firefly Optimized Lottery Scheduling (NMT-FOLS) Technique outperforms existing state of art and conventional scheduling algorithms.

\section{References}

1. Balamurugan, M. S., \& Manojkumar, R. (2019). Study of short term rain forecasting using machine learning based approach. Wireless Networks. https://doi.org/10.1007/s11276-019-02168-3

2. Sudhakar, M., \& Meena, M. J. (2019). An efficient interactive segmentation algorithm using color correction for underwater 
images. Wireless Networks. https://doi.org/10.1007/s11276-01902044-0

3. Devika, R., \& Subramaniyaswamy, V. (2019). A semantic graphbased keyword extraction model using ranking method on big social data. Wireless Networks. https://doi.org/10.1007/s11276019-02128-X

4. Jain, U., Mishra, A., Jaganathan, B., et al. (2021). Study and analysis of category based PageRank method. Wireless Networks. https://doi.org/10.1007/s11276-021-02617-y

5. Thillaiarasu, N., Pandian, S. C., Vijayakumar, V., et al. (2019). Designing a trivial information relaying scheme for assuring safety in mobile cloud computing environment. Wireless Networks. https://doi.org/10.1007/s11276-019-02113-4

6. Dhanapal, A., \& Nithyanandam, P. (2019). An OpenStack based cloud testbed framework for evaluating HTTP flooding attacks. Wireless Networks. https://doi.org/10.1007/s11276-019-01937-4

7. Somasundaram, R., \& Thirugnanam, M. (2020). Review of security challenges in healthcare internet of things. Wireless Networks. https://doi.org/10.1007/s11276-020-02340-0

8. Shanmugam, R. (2019). Parity between prescription and visitation rates in healthcare. Wireless Networks. https://doi.org/10. 1007/s11276-019-02204-2

9. Padma Priya, R., \& Rekha, D. (2020). Sustainability modelling and green energy optimisation in microgrid powered distributed FogMicroDataCenters in rural area. Wireless Networks. https:// doi.org/10.1007/s11276-019-02207-z

10. Anusooya, G., \& Vijayakumar, V. (2019). Reduced carbon emission and optimized power consumption technique using container over virtual machine. Wireless Networks. https://doi. org/10.1007/s11276-019-02001-x

11. Karmel, A., Vijayakumar, V., \& Kapilan, R. (2019). Ant-based efficient energy and balanced load routing approach for optimal path convergence in MANET. Wireless Networks. https://doi.org/ 10.1007/s11276-019-02080-w

12. Joshua, C. J., \& Varadarajan, V. (2019). An optimization framework for routing protocols in VANETs: A multi-objective firefly algorithm approach. Wireless Networks. https://doi.org/10. 1007/s11276-019-02072-w

13. Rajarajeswari, S., \& Neelanarayanan, V. (2019). Dynamic rule generation and rule matching for composite environmental condition in context aware system. Wireless Networks. https://doi. org/10.1007/s11276-019-02081-9

14. Muralidharan, C., \& Anitha, R. (2019). Risk analysis of cloud service providers by analyzing the frequency of occurrence of problems using E-Eclat algorithm. Wireless Networks. https://doi. org/10.1007/s11276-019-02191-4

15. Prassanna, J., \& Venkataraman, N. (2019). Adaptive regressive holt-winters workload prediction and firefly optimized lottery scheduling for load balancing in cloud. Wireless Networks. https://doi.org/10.1007/s11276-019-02090-8

Publisher's Note Springer Nature remains neutral with regard to jurisdictional claims in published maps and institutional affiliations.

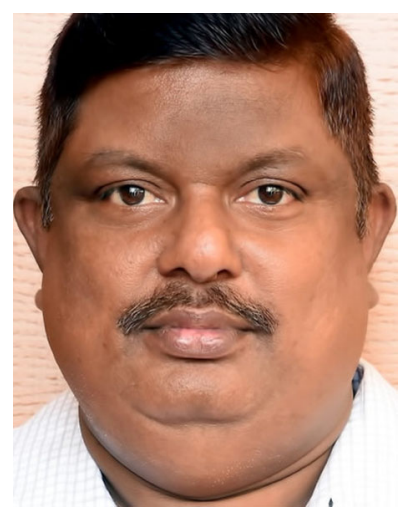

V. Neelanarayanan received his Master of Science in Computer Science from Madurai Kamaraj University, India in 1995 and PhD from IT University of Copenhagen, Denmark in 2012. Currently, he is an Associate Professor Senior and Head, Department of Cyber Physical Systems at VIT University, Chennai, India. Before joining VIT University he has worked as a Scientist at Centre for Advanced Computing (CDAC), India and as a Lecturer in Madurai Kamaraj University, India and its affiliated institutions. His areas of research include distributed computing such as grid, cloud and fog computing, context-aware computing, network management and security, XML-based security technologies and e-communities. He received the research award in VIT University for the year 2015 and 2018 for his achievements, exemplary commitment, dedication and motivation towards research publication during 2015-16 and 2018-19 respectively. He has published five books and more than 50 papers in various peer-reviewed international conferences and journals. He has published 8 indian patenst. He has organized various national workshops, international conferences and international symposiums. According to bookauthority, his book on cloud computing is one among top 10 cloud computing ebooks in 2020. He has served as editor-in-chief for EAI endorsed transaction in cloud systems from $2018-20$. He is also a member of several national and international professional bodies including ISTE, IAENG, CSTA, etc.

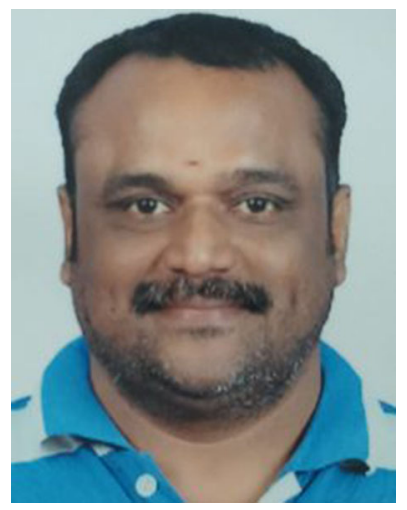

V. Vijayakumar is currently a Adjunct Professor, School of Computer Science and Engineering, The University of New South Wales, Sydney, Australia. Earlier he served as a Professor and an Associate Dean for School of Computing Science and Engineering at VIT University, Chennai, India. He also served as a Team Lead in industries like Satyam, Mahindra Satyam and Tech Mahindra for several years. He has completed Diploma with First Class Honors. He has completed BE CSE and MBA HRD with First Class. He has also completed ME CSE with First Rank Award. He has completed his PhD from Anna University in 2012. He has published many articles in national and international level journals/ conferences/books. He has initiated a number of international research collaborations with universities in Europe, Australia, Africa and North America including University of Missouri. He had also initiated joint research collaboration between VIT University and industries. $\mathrm{He}$ also organised several international conferences and special sessions in USA, Vietnam, Africa and India including IEEE, ISBCC etc. His research interests include computational areas covering grid computing, cloud computing, computer networks and big data. He received his university-level Best Faculty Award for 2015-2016 year at VIT University. He is also a member of several national and international. 


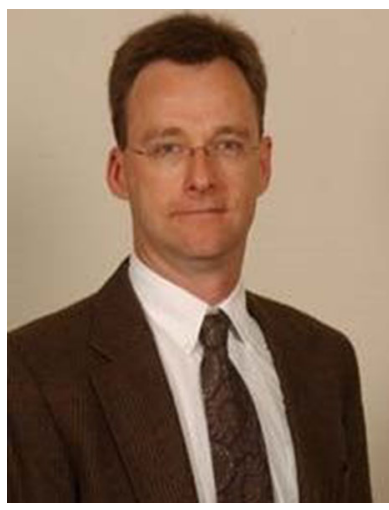

Dr. Ron Doyle is the Katherine and Dickerson Wright Presidential Chair in Computer Science and Entrepreneurship at Wake Forest University. Dr. Doyle is also the CTO of the Enterprise Software Division at Broadcom, serving as VP of Architecture for the Continuous Delivery business unit at CA Technologies prior to acquisition. Previously, he was a Distinguished Engineer in the IBM Cloud division. He holds a $\mathrm{PhD}$ in Computer Science from

Duke University.

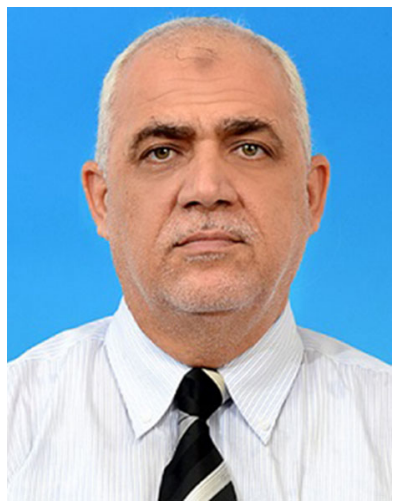

Prof . Dr. Imad Fakhri Taha Alyaseen he is a full professor, currently work in Kulliyyah of Information and Communication Technology. He received $\mathrm{Ph} . \mathrm{D}$. degree in Computer Science from University of Pune, India in 2000, and Master degree in Computer Science from University of Technology, Iraq. His Bachelor Degree in Mathemathics, Al-Mustansiriya University, Iraq. He is an IEEE senior member. In 2003, he was appointed as the Head of Department of Computer Information Systems at Alrafidain University College until 2005. Then he joined Gulf University- Bahrain in January 2006 and was appointed as the founding Dean of the College of Computer Engineering and Sciences.During this period, he introduced the CCNA certificate to be part of the curriculum which had a strong impact on the student's careers. In November 2010, he joined IIUM at the Dept. of Computer Science / Kulliyyah of Information and Communication Technology. He received the Best Teacher Award in 2011. He has been the editor in chief of International journal on Advanced Computer Science and Technology Research since 2011 now, and the general chair of the international conference on Advanced Computer Science Applications and Technologies since 2012 till now. He obtained a US patent for his work with his Ph.D. student on smart traffic light with accident detection system on 2nd December 2014. Prof. Dr. Imad has published more than 100 papers in, journals and book chapters in addition to three books.

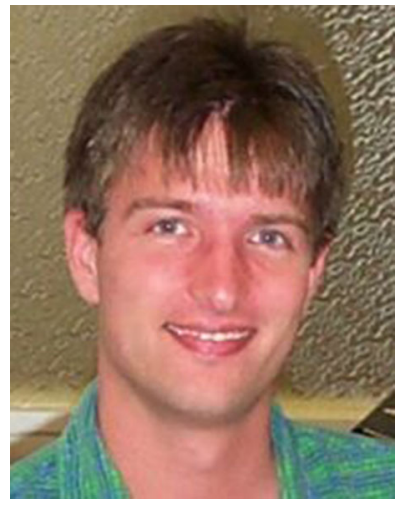

Dr. Sven Groppe is a Lecturer and Tutor at the University of Lübeck, Germany. His publication record contains over 100 publications, including the book Data Management and Query Processing in Semantic Web Databases, published by Springer. He was a member of the DAWG W3C Working Group, which developed SPARQL. He was the project leader of the DFG project LUPOSDATE, an open-source Semantic Web database, and of two research projects in the area of FPGA acceleration of relational and Semantic Web databases. He is also leading a DFG project on GPU and APU acceleration of main-memory database indexes, and a DFG project about Semantic Internet of Things. He is also the chair of the Semantic Big Data workshop series, which is affiliated with the ACM SIGMOD conference (so far 2016 to 2020), and of the Very Large Internet of Things workshop in conjunction with the VLDB conference (so far 2017 to 2020). He is general chair of the upcoming International Semantic Intelligence Conference (ISIC) in 2021. His research interests include artificial intelligence, databases, Semantic Web, query and rule processing and optimization, Cloud Computing, acceleration via GPUs and FPGAs, peer-to-peer (P2P) networks, Internet of Things, data visualization and visual query languages. 